\title{
Expression of hMOF in different ovarian tissues and its effects on ovarian cancer prognosis
}

\author{
MINGBO CAI, ZHENHUA HU, JUANJUAN LIU, JIAN GAO, MINGZI TAN, DANYE ZHANG, \\ LIANCHENG ZHU, SHUICE LIU, RUI HOU and BEI LIN
}

Department of Obstetrics and Gynecology, Shengjing Hospital Affiliated to China Medical University, Heping, Shenyang, Liaoning 110004, P.R. China

Received October 3, 2014; Accepted November 7, 2014

DOI: $10.3892 /$ or.2014.3649

\begin{abstract}
Human MOF (hMOF) is a major acetylase of human H4K16 involved in the regulation of physiological and pathological processes. We investigated the expression of hMOF in different ovarian tissues and its correlation with ovarian cancer prognosis. Reverse transcription PCR and western blot analysis were used to detect hMOF mRNA and protein expression, respectively, in different ovarian tissues. Immunohistochemistry was also performed to detect hMOF expression in different ovarian tissues, including ovarian epithelial cancer, borderline tumor, benign tumor and normal ovarian tissues. In addition, the relationships between hMOF expression and clinicopathological ovarian cancer data were analyzed. The Cox proportional-hazards regression model was used to analyze the factors associated with ovarian cancer prognosis. To analyze the effects of hMOF expression on ovarian cancer prognosis, a survival curve was plotted from the follow-up data of 77 patients with ovarian cancer. Compared with normal ovarian tissues, hMOF mRNA and protein expression was significantly decreased in ovarian epithelial cancer tissues. The proportions of high hMOF expression in normal and benign ovarian epithelial tumor tissues, were much higher than those in ovarian epithelial cancer tissues. Furthermore, hMOF protein expression was closely associated with the ovarian cancer stage. The expression of hMOF protein was determined as an independent risk factor influencing ovarian cancer prognosis. Patients with high hMOF levels showed improved survival than those with low hMOF levels. hMOF mRNA and protein expression decreased
\end{abstract}

Correspondence to: Dr Bei Lin, Department of Obstetrics and Gynecology, Shengjing Hospital Affiliated to China Medical University, No. 36 Sanhao Street, Heping, Shenyang, Liaoning 110004, P.R. China E-mail: linbei88@hotmail.com

Key words: histone acetyltransferase, hMOF, ovarian cancer, prognosis, survival analysis in ovarian epithelial cancer, thus the hMOF protein potentially serves as a new clinical marker of ovarian cancer prognosis.

\section{Introduction}

Histone acetylation modification is an important epigenetic modification under the dual regulation of histone acetyltransferases (HATs) and histone deacetylases (HDACs) (1). HATs are important in the regulation of genetic transcription, chromosome constitution, DNA damage repair and cell cycle regulation (2-4). It is closely associated with the occurrence and development of several diseases such as cancer, neurodegeneration and inflammatory lung disease (5-7). The p300 acetyltransferase serves as a tumor suppressor and is mutated in epithelial malignancies (8). HDAC1 is significantly increased in hormone-refractory prostate cancer cell lines and promotes the malignant transformation of cancer cells (9).

HATs are classified as: GNAT family (Gcn5-related-Nacetyltransferase family), p300 (E1A-associated protein of $300 \mathrm{kDa}$ ) or CBP (CREB-binding protein) and MYST family members (10). As a MYST family member, males absent on the first (MOF) is part of the dosage compensation complex on the X chromosome of male Drosophila and an important component of the male-specific lethal complex $(11,12)$. MOF impacts several physiological and pathological processes such as mammalian embryonic development, the maintenance of chromatin structure, and tumorigenesis. MOF influences the chromatin structure of mouse embryonic cells and the implantation of early embryos (13). MOF also plays an essential role in the maintenance of embryonic stem cell self-renewal and pluripotency (14).

Human MOF (hMOF) and Drosophila MOF are orthologous, and hMOF specifically acetylates the histone H4K16 (15). The substrate specificity of hMOF depends on its formation of different complexes. For example, hMOF containing NSL complex can acetylate histone H4K16, H4K5 and H4K8 (16). When the hMOF gene is knocked down, H4K16 acetylation levels significantly decrease $(15,17)$. hMOF participates in basic physiological processes of mammalian cells, including gene transcription, cell proliferation and differentiation, nuclear morphology, chromatic constitution and DNA repair (18). Therefore, the inactivation of hMOF may be an important step in the malignant transformation of cells. 
After the knockdown of the $h M O F$ gene expression in HeLa and HepG2 cells, nuclear morphology obviously changed into a multi-leaved shape (17). However, whether the nuclear morphological change was the result or cause of the cellular malignant transformation remains to be determined.

The role of hMOF in the occurrence, development and prognosis of malignant tumors has been investigated. Pfister et al (19) found that hMOF and H4K16 are frequently downregulated in primary breast carcinoma and medulloblastoma. Furthermore, they found that hMOF protein expression is a prognostic marker for medulloblastoma, with patients harboring tumors with a low hMOF expression having a significantly worse survival (19). In a study on human renal cell carcinoma, Wang et al found that the expression of hMOF in cancer tissues was significantly lower than that in adjacent kidney tissues (20). However, hMOF was more frequently found to be highly expressed in non-small cell lung cancer (NSCLC) than corresponding normal tissues, and hMOF promoted the proliferation, metastasis and adhesion of NSCLC cell lines (21)

Although the low expression of hMOF in ovarian cancer has been identified (22), the relationship between the hMOF expression and ovarian cancer prognosis has yet to be reported. hMOF may be important in the occurrence, development and prognosis of ovarian cancer. Thus, the expression of hMOF in different ovarian tissues and its relationship with ovarian cancer prognosis were investigated.

\section{Materials and methods}

Patients and follow-up. Experiments were approved by the Institutional Review Board of Shengjing Hospital at China Medical University. A total of 45 samples were collected from tissues removed during the surgical removal of cervical cancers at the Department of Gynecology, Shengjing Hospital, China. The 45 samples comprised 30 samples of ovarian epithelial cancer tissues and 15 samples of normal ovarian tissues. All the tissues were removed during surgery whereupon they were immediately frozen in liquid nitrogen and stored at $-80^{\circ} \mathrm{C}$. Based on the characteristics of ovarian carcinoma, it is extremely difficult to obtain para-carcinoma tissue; therefore, normal ovarian tissues were used as a control group. Patients anonymity was maintained. The tissue samples were examined by specialists to obtain a final diagnosis, and no patients were administered chemotherapy or radiotherapy prior to surgery. The clinicopathological characteristics of 30 patients of epithelial ovarian cancer are shown in Table I.

In total, 45 fresh tissue samples were embedded into paraffin sections and an additional 136 different paraffin slices were obtained from the ovarian tissues resected during operations at the Department of Gynecology, Shengjing Hospital, China Medical University, China, from 2008 to 2012. A total of 181 paraffin sections were thus obtained: 112 of primary malignant ovarian tumors, 23 of borderline ovarian tumors, 26 of benign ovarian tumors and 20 of normal ovarian tissues. The tissue sections were examined by experienced specialists to obtain a final diagnosis. Histopathological diagnoses were made using the World Health Organization criteria. The classification of cancer stage and grade was carried out according to the International Federation of Gynecology and Obstetrics.
Table I. Clinicopathological characteristics of 30 patients with ovarian cancer.

\begin{tabular}{lr}
\hline Characteristics & No. \\
\hline Pathologic type & 20 \\
Serous & 2 \\
Mucinous & 1 \\
Clear cell carcinoma & 7 \\
Poorly differentiated adenocarcinoma & \\
Surgical stage & 9 \\
I-II & 21 \\
III-IV & \\
Differentiation & 5 \\
Well & 25 \\
Moderate-poor & \\
Lymph node metastasis & 27 \\
No & 3 \\
Yes & \\
\hline
\end{tabular}

The clinical and pathological information concerning the patients was collected from their clinical records, including their age, surgical stage, lymph node metastasis, pathological tumor grade and subtype, and residual tumor size.

The age range (median) was $16-77$ years ( 52.7 years) in the malignant ovarian tumor group; $21-78$ years ( 41.5 years) in the borderline ovarian tumor group; $24-61$ years (43.2 years) in the benign ovarian tumor group; and 45-68 years (58.9 years) in the normal ovarian tissue group. There were no statistically significant differences in the ages of these groups $(\mathrm{P}>0.05)$.

Information was collected on the clinical chemotherapeutic treatments received and the follow-up from 77 patients out of a total 112 patients with malignant ovarian cancer (the information from 77 patients was complete and these 77 patients were followed-up for 24 months at least after surgery). These 77 patients underwent treatments for ovarian cancer that included surgical debulking followed by $6-8$ postoperative cycles of conventional chemotherapy, consisting of paclitaxel (Yangtze River Pharmaceutical Group, Taizhou, China) and carboplatin (Qilu Pharmaceutical Co., Ltd, Jinan, China). Overall survival (OS) was defined as the date of surgery to the date of death or the last follow-up. Disease-free survival (DFS) was defined as the interval from the initial surgery to clinically or radiologically proven recurrence/metastasis and deceased. Following surgery, the patients were observed at 3-month intervals. As of May 2014, it has been 70 months since the first patient was recruited into the research group, and it has been 24 months since the last patient was recruited into our group. The median followup period was 48 months. To determine the factors influencing survival after surgery and standard chemotherapy, conventional variables, together with hMOF expression, were assessed in 77 ovarian carcinoma patients.

Materials. The PrimeScript ${ }^{\mathrm{TM}}$ 1st Strand cDNA Synthesis and PCR amplification kits were purchased from Takara (6110A, RR02A; Dalian, China). Mouse monoclonal anti-hMOF 
antibody (GTX83065) was obtained from GeneTex (Irvine, CA, USA). The anti-GAPDH (glyceraldehyde-3-phosphate dehydrogenase) monoclonal antibody was purchased from Boshide Biotech (BM1985; Wuhan, China).

Reverse transcription PCR (RT-PCR). Total RNA from epithelial ovarian cancer and the normal ovarian tissues were isolated using TRIzol ${ }^{\circledR}$ LS reagent (Invitrogen, Carlsbad, CA, USA). RNA (500 ng) from each sample was used as a template to produce cDNA using the PrimeScript ${ }^{\mathrm{TM}}$ 1st Strand cDNA Synthesis kit. PCR reactions were performed under the following conditions: an initial denaturation step at $95^{\circ} \mathrm{C}$ for $2 \mathrm{~min}$, followed by 30 cycles of denaturation step at $95^{\circ} \mathrm{C}$ for $30 \mathrm{sec}$, annealing at $57^{\circ} \mathrm{C}$ for $30 \mathrm{sec}$ and extension at $72^{\circ} \mathrm{C}$ for $30 \mathrm{sec}$. The primer sets used for the PCR were: GAPDH, forward: 5'-ATCACTGCCACCCAGAAGAC-3' and reverse: 5'-ATGAGGTCCACCACCCTGTT-3', yielding a 433-bp product; hMOF, forward: 5'-GACACTGTACTTTGACGTGG AGC-3' and reverse: 5'-CACTGTGATGGGTGGTTTCTT-3', yielding a 493-bp product.

Immunoblotting. Soluble proteins were isolated from tissues for western blotting. The protein concentrations were measured by bicinchoninic acid (23228; Thermo Fisher Scientific, Waltham, MA, USA). Equal amounts of protein from each sample were separated by electrophoresis on an SDS-10\% polyacrylamide gel, transferred to a polyvinylidene difluoride membranes, and blocked with $5 \%$ non-fat dry milk in $1 \mathrm{X}$ TBS plus $0.1 \%$ Tween-20 at room temperature for $2 \mathrm{~h}$. The membranes were incubated overnight at $4^{\circ} \mathrm{C}$ with primary antibodies in $1 \%$ bovine serum albumin in $1 \mathrm{X}$ TBS plus $0.1 \%$ Tween20. The primary anti-hMOF monoclonal antibody (diluted 1:1,000) was purchased from GeneTex (GTX83065) and the anti-GAPDH monoclonal antibody (diluted 1:2,000) was purchased from Boshide Biotech (BM1985). Membranes were washed and incubated again for $2 \mathrm{~h}$ at room temperature with horseradish-peroxidase-conjugated anti-mouse secondary antibodies. Proteins were visualized with ECL reagent (ECL Prime Western Blotting Detection Reagent; Amersham, Pittsburgh, PA, USA). The experiments were repeated three times.

Immunohistochemistry. Paraffin-embedded histological sections from each group of ovarian tissues were cut into $5-\mu \mathrm{m}$ slices. Immunohistochemistry was used to analyze the hMOF protein expression levels. Mouse monoclonal anti-hMOF antibody (GTX83065; diluted 1:800) was purchased from the GeneTex. The staining procedure was performed according to the manual of an ultrasensitive streptavidin-peroxidase kit (KIT-9701; Maixin Bio, Fuzhou, China) and Harris's hematoxylin was used to stain the cell nuclei. Tissues were treated with phosphate-buffered saline instead of primary antibody as a negative control. Buff-colored granules in the cell nucleus were considered a positive result. The tissues were rated according to their chromatic intensity: no pigmentation, 0 ; light yellow, 1 ; buff, 2 ; and brown, 3 . Five high-power fields in serial sections from each slice were selected, scored, and the mean percentage of chromatic cells was estimated: $<5 \%$ chromatic cells, $0 ; 5-25 \%$ chromatic cells, $1 ; 26-50 \%$ chromatic cells, 2 ; $51-75 \%$ chromatic cells, 3 ; and $>75 \%$ chromatic cells, 4 . The two numbers (intensity score $\mathrm{x}$ percentage chromatic cells)
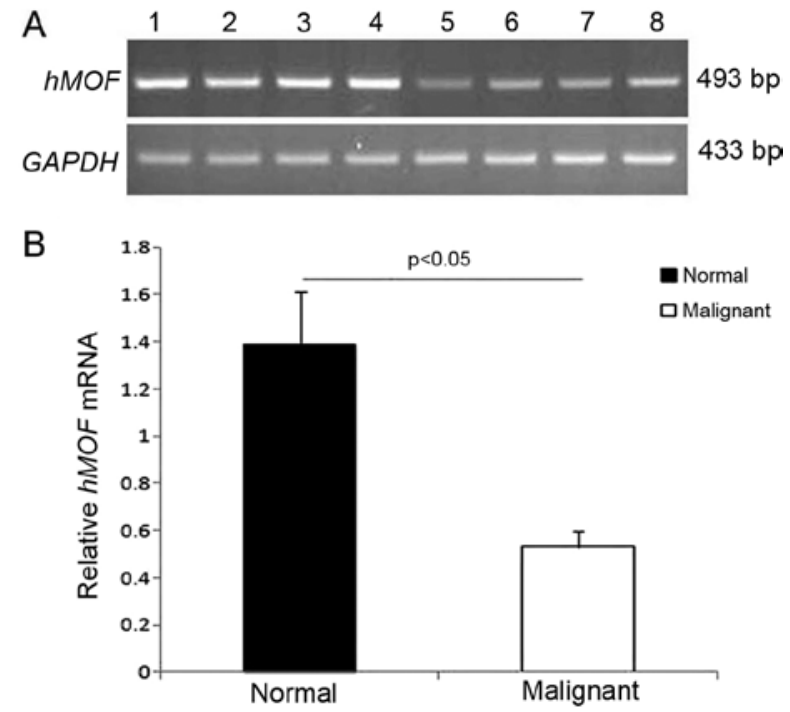

Figure 1. The expression of $h M O F$ mRNA in normal ovarian and ovarian cancer tissues. (A) RT-PCR indicates $h M O F$ mRNA expression is elevated in normal ovarian tissues compared with ovarian cancer tissues. Lanes 1-4, normal ovarian tissues; lanes 5-8, ovarian cancer tissues. The different lines represent different samples. (B) Quantitative analysis of $h M O F$ mRNA expression in normal ovarian tissues (15 cases) and ovarian cancer tissues (30 cases). The expression of $h M O F$ mRNA is decreased in ovarian cancer tissues compared with normal ovarian tissues $(\mathrm{P}<0.05)$. hMOF, human MOF; MOF, males absent on the first.

were multiplied and scored: 0-2 was considered (-), 3-4 (+), 5-8 $(++)$ and 9-12 (+++). The 112 patients of ovarian cancer were divided into the high hMOF expression group $(++/+++)$ and the low hMOF expression group (-/+). Two observers read the sections to control for systemic error.

Statistical analyses. The gene expression and western blot images were scanned and quantified with Image J software (National Institutes of Health, Bethesda, MD, USA). Differences in proportions were evaluated using the $\chi^{2}$ test. The $\chi^{2}$ test or Fisher's exact test, whichever was appropriate, was used to analyze the relationship between hMOF expression and clinicopathological variables. A survival curve was generated using the Kaplan-Meier method and compared by the log-rank test. Cox's proportional hazard regression model was used for multivariate survival analysis of prognostic factors. Statistical analyses were performed using SPSS v17.0 software (SPSS, Inc., Chicago, IL, USA). The Student's two-tailed t-test was used in all analyses. $\mathrm{P}<0.05$ was considered to indicate a statistically significant result.

\section{Results}

Reduced hMOF mRNA expression in ovarian cancer. $\mathrm{hMOF}$ plays a role in the basic physiological processes of mammalian cells and therefore, it may be involved in different malignant tumors. Consequently, we hypothesized that hMOF may be important in the occurrence and development of ovarian cancer and that the role of hMOF may differ between ovarian cancer and normal ovarian tissues. RT-PCR showed that the expression of hMOF mRNA in normal ovarian tissues (15 cases) was 2.62-fold higher than that in ovarian cancer tissues (30 cases) $(\mathrm{P}<0.05$; Fig. $1 \mathrm{~A}$ and $\mathrm{B})$. 
Table II. hMOF in different ovarian tissues.

\begin{tabular}{|c|c|c|c|c|c|c|c|c|}
\hline \multirow[b]{2}{*}{ Groups } & \multirow[b]{2}{*}{ Cases } & \multicolumn{4}{|c|}{ hMOF expression } & \multirow[b]{2}{*}{ Positive rates (\%) } & \multicolumn{2}{|c|}{ hMOF expression } \\
\hline & & - & + & ++ & +++ & & Low $(-/+)$ & High $(++/+++)$ \\
\hline Malignant & 112 & 22 & 59 & 16 & 15 & 80.36 & $81(72.32 \%)$ & $31(27.68 \%)^{\mathrm{a}}$ \\
\hline Borderline & 23 & 8 & 8 & 3 & 4 & 65.22 & $16(69.57)$ & $7(30.43 \%)$ \\
\hline Benign & 26 & 4 & 7 & 6 & 9 & 84.62 & $11(42.31 \%)$ & $15(57.69 \%)$ \\
\hline Normal & 20 & 4 & 6 & 4 & 6 & 80.00 & $10(50.00 \%)$ & $10(50.00 \%)$ \\
\hline
\end{tabular}

${ }^{\mathrm{a} C}$ Compared with the benign $(57.69 \%)$ or normal group (50.00\%). $\mathrm{P}<0.05 . \mathrm{hMOF}$, human MOF; MOF, males absent on the first.
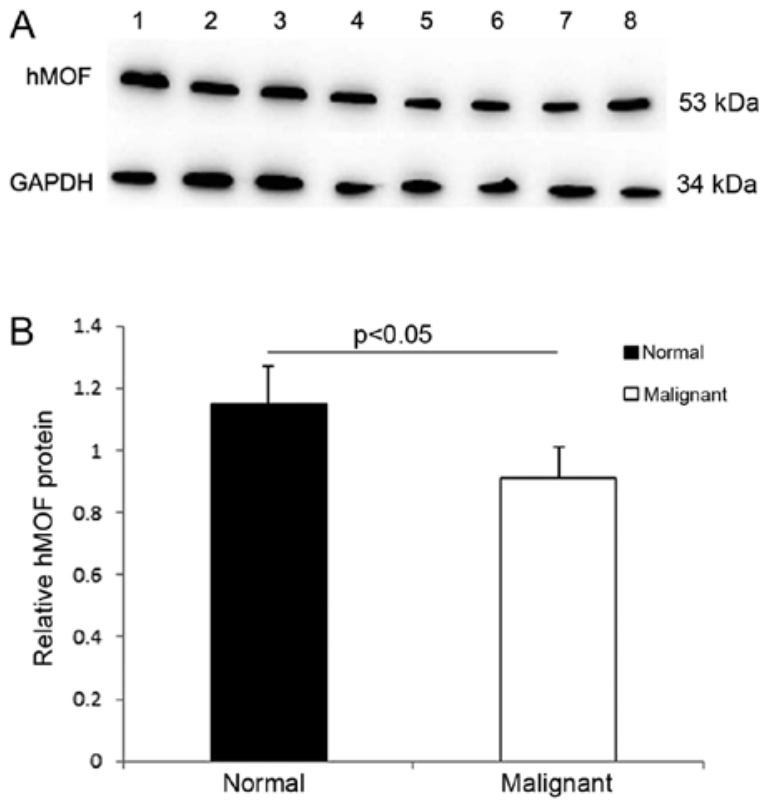

Figure 2. The expression of hMOF protein in normal ovarian and ovarian cancer tissues. (A) Western blot analysis reveals hMOF protein expression is elevated in normal ovarian tissues compared to ovarian cancer tissues. Lanes 1-4, normal ovarian tissues; lanes 5-8, ovarian cancer tissues. The different lines represent different samples. (B) Quantitative data of hMOF protein expression in normal ovarian tissues (15 cases) and ovarian cancer tissues (30 cases). The expression of hMOF protein is decreased in ovarian cancer tissues compared to normal ovarian tissues $(\mathrm{P}<0.05)$. hMOF, human MOF; MOF, males absent on the first.

Reduced hMOF protein expression in ovarian cancer. The results from the western blot analysis and immunohistochemical methods used to investigate hMOF protein expression in different ovarian tissues, confirmed the results from the mRNA expression analysis. Western blot analysis showed that hMOF protein expression in the normal ovarian tissues was 1.28-fold higher than that in ovarian cancer tissues $(\mathrm{P}<0.05$; Fig. 2A and B).

Immunohistochemical analysis of hMOF protein expression in different ovarian tissues. hMOF immunoreactivity was identified in the nucleus (Fig. 3) and in all tissue types, and hMOF was expressed in the nucleus. The positive hMOF protein expression rates in ovarian epithelial cancer, borderline tumor, benign tumor and normal ovarian tissues were $80.36,65.22,84.62$ and $80.00 \%$, respectively (Table II). The
Table III. Relationships between hMOF protein expression and clinicopathological characteristics of 112 patients with malignant ovarian cancer.

\begin{tabular}{|c|c|c|c|c|}
\hline \multirow[b]{2}{*}{ Characteristics } & \multirow[b]{2}{*}{$\begin{array}{l}\text { No. } \\
\text { cases }\end{array}$} & \multicolumn{2}{|c|}{ hMOF expression } & \multirow[b]{2}{*}{ P-value } \\
\hline & & $\begin{array}{c}\text { Low } \\
(-/+) \\
(\%)\end{array}$ & $\begin{array}{c}\text { High } \\
(++/+++) \\
(\%)\end{array}$ & \\
\hline Pathological type & & & & $<0.05$ \\
\hline Serous & 48 & $37(77.08)$ & $11(22.92)^{\mathrm{a}}$ & \\
\hline Mucinous & 19 & $13(68.42)$ & $6(31.58)$ & \\
\hline Endometrioid & 8 & $3(37.50)$ & $5(62.50)$ & \\
\hline $\begin{array}{l}\text { Clear cell } \\
\text { carcinoma }\end{array}$ & 10 & $8(80.00)$ & $2(20.00)$ & \\
\hline $\begin{array}{l}\text { Poorly } \\
\text { differentiated } \\
\text { adenocarcinoma }\end{array}$ & 27 & $20(74.07)$ & 7 (25.93) & \\
\hline Surgical stage & & & & $<0.05$ \\
\hline I & 36 & $24(66.67)$ & $12(33.33)^{\mathrm{b}}$ & \\
\hline II & 13 & $11(84.61)$ & $2(11.39)$ & \\
\hline III & 57 & $40(70.18)$ & $17(29.82)$ & \\
\hline IV & 6 & $6(100.00)$ & $0(0.00)$ & \\
\hline Differentiation & & & & $>0.05$ \\
\hline Well & 16 & $10(62.50)$ & $6(37.50)$ & \\
\hline Moderate & 30 & $24(80.00)$ & $6(20.00)$ & \\
\hline Moderate-poor & 19 & $13(68.42)$ & $6(31.58)$ & \\
\hline Poor & 47 & $34(72.34)$ & $13(27.66)$ & \\
\hline $\begin{array}{l}\text { Lymph node } \\
\text { metastasis }^{c}\end{array}$ & & & & $>0.05$ \\
\hline No & 90 & $62(68.89)$ & $28(31.11)$ & \\
\hline Yes & 17 & $14(82.35)$ & $3(17.65)$ & \\
\hline
\end{tabular}

${ }^{\mathrm{a} C}$ Compared with the endometrioid group, $\mathrm{P}<0.05$. ${ }^{\mathrm{b} C o m p a r e d ~ w i t h ~ t h e ~}$ IV group, $\mathrm{P}<0.05$. ${ }^{\mathrm{c}}$ Five cases of ovarian carcinoma patients without lymph-node resection. hMOF, human MOF; MOF, males absent on the first.

positive rates did not significantly differ between groups. Data were divided into a low hMOF expression group (including negative and weak positive expression) and a high hMOF 

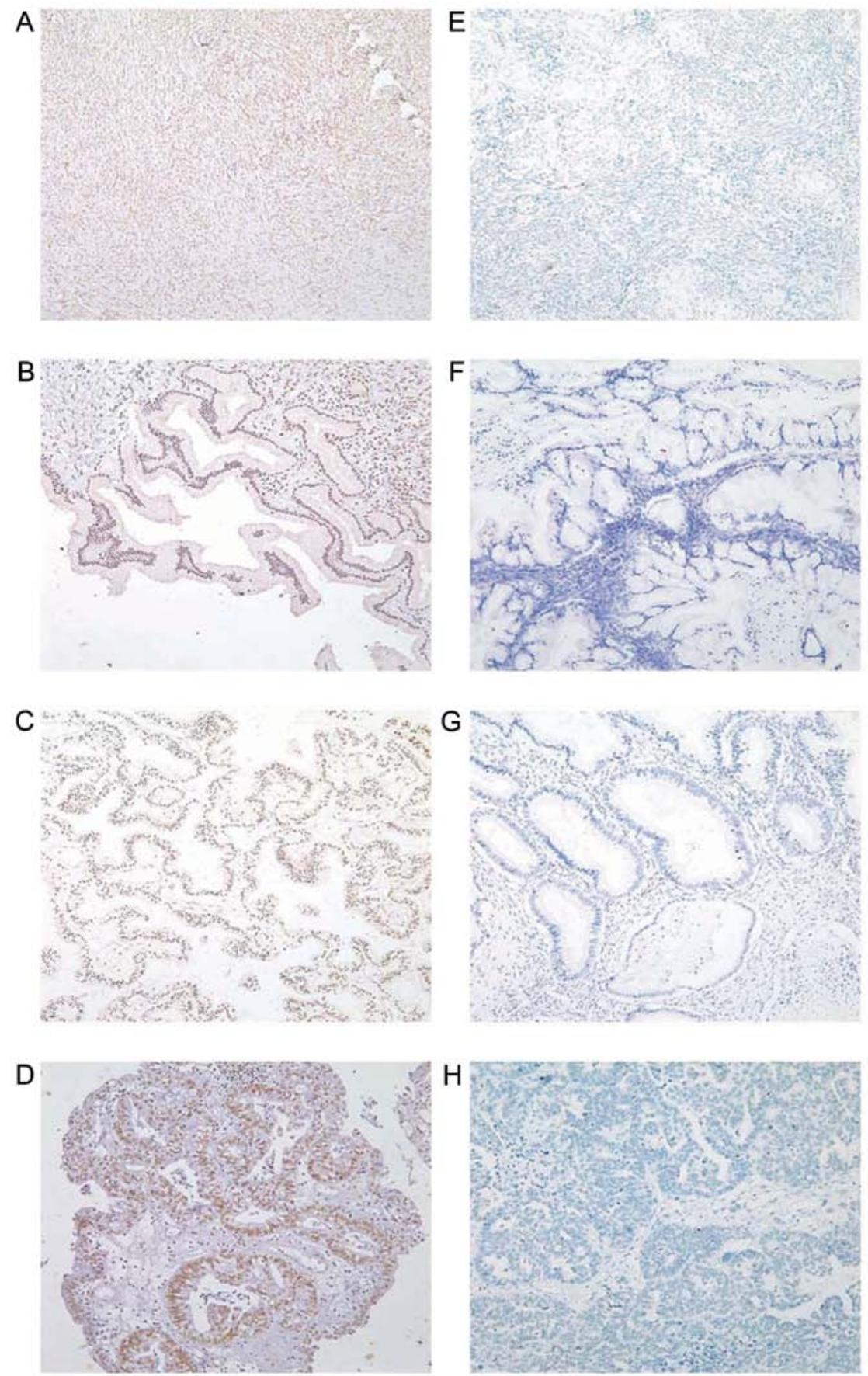

Figure 3. Immunohistochemical micrographs of hMOF protein expression in different ovarian tissues (x200). (A and E) Normal ovarian tissues; (B and F) benign; (C and G) borderline; (D and H) malignant. (A-D) hMOF is highly expressed in different ovarian tissues; (E-H) hMOF has a low expression in different ovarian tissues. hMOF, human MOF; MOF, males absent on the first.

expression group (including moderate and strong positive expression). According to the standard scoring of paraffin slices, 3-4 was defined as weak expression and 5-8 was defined as moderate expression. The specific scoring criteria was as described in Materials and methods. Further analysis of the data found that the proportions of high hMOF expression in ovarian epithelial cancer, borderline tumor, benign tumor and normal ovarian tissues, were 27.68, 30.43, 57.69 and $50.00 \%$, respectively (Table II). Therefore, hMOF protein expression in ovarian benign tumor tissues and normal ovarian tissues was much higher than that in ovarian epithelial cancer tissues $(\mathrm{P}<0.05)$.
Relationship between hMOF expression and clinicopathological data on ovarian cancer. A total of 112 ovarian cancer patients were divided into the high $(++/+++)$ and low (-/+) hMOF protein-expression groups. The rate of high hMOF protein expression in stage I ovarian cancer tissues was $33.33 \%$, significantly higher than its expression in stage IV ovarian cancer tissues $(\mathrm{P}<0.05)$. The protein expression of hMOF in ovarian endometrioid adenocarcinoma tissues was significantly higher than its expression in ovarian serous adenocarcinoma tissues $(\mathrm{P}<0.05)$. There was no relationship between hMOF protein expression with cell differentiation and lymph node metastasis $(\mathrm{P}>0.05$; Table III). 
Table IV. Cox proportional hazards regression model.

\begin{tabular}{lccrrr}
\hline & \multicolumn{2}{c}{ Overall survival (OS) } & & \multicolumn{2}{c}{ Disease-free survival (DFS) } \\
\cline { 2 - 3 } Variables & Hazard ratio (95\% CI) & P-value & & Hazard ratio (95\% CI) & P-value \\
\hline hMOF & $0.337(0.125-0.903)$ & 0.031 & & $0.307(0.114-0.829)$ & 0.020 \\
Age (years) & $0.381(0.158-0.919)$ & 0.032 & & $0.451(0.190-1.067)$ & 0.070 \\
Surgical stage & $1.588(0.657-1.255)$ & 0.558 & & $1.820(0.701-4.729)$ & 0.219 \\
Differentiation & $1.015(0.390-2.642)$ & 0.976 & & $1.073(0.426-2.706)$ & 0.881 \\
Pathological type & $0.908(0.657-1.255)$ & 0.705 & & $0.938(0.673-1.307)$ & 0.705 \\
Lymph node metastasis & $1.905(0.724-5.012)$ & 0.191 & & $2.394(0.876-6.545)$ & 0.089 \\
Residual lesion size & $1.719(0.953-3.100)$ & 0.072 & & $1.877(1.029-3.461)$ & 0.040 \\
\hline
\end{tabular}

CI, confidence interval. hMOF, human MOF; MOF, males absent on the first.
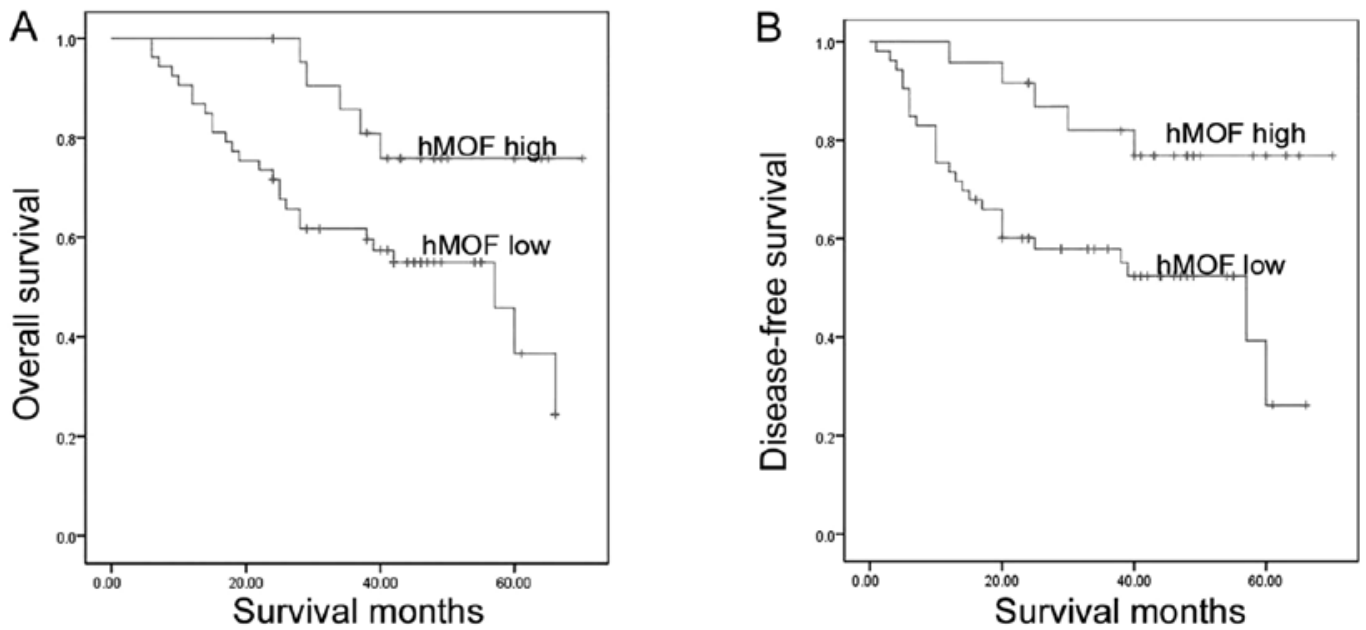

Figure 4. The association among overall and disease-free survival, and hMOF protein expression in 77 patients with ovarian cancer. (A) The overall survival rate and (B) disease-free survival rate were significantly higher for those patients in the high hMOF-expression group compared to the low hMOF expression group (both $\mathrm{P}<0.05$ ). hMOF, human MOF; MOF, males absent on the first.

Multivariate prognostic analysis of ovarian cancer. A total of 77 patients with complete follow-up data were divided into the low (-/+) and high (++/+++) hMOF protein-expression groups. The COX proportional-hazards regression model was applied with survival time as an independent variable and dependent variables including the age of ovarian cancer patients, surgical pathological staging, differentiation, pathological category, lymph-node metastasis, residual tumor size and hMOF expression. Multivariate analysis revealed that the age of ovarian cancer patients and hMOF expression were independent prognostic risk factors for OS (Table IV), while residual tumor size and hMOF expression were the independent risk factors closely associated with DFS (Table IV).

Comparison of survival rate of ovarian cancer patients. As of May 2014 (70 months since the recruitment of the first patient and 24 months since recruitment of the last patient), 26 patients in the low hMOF-expression group (comprising 53 patients) succumbed, while five patients in the high hMOF-expression group (comprising 24 patients) succumbed. A Kaplan-Meier analysis (Fig. 4) showed that the OS and DFS rates were signif- icantly higher in the high hMOF expression group compared to the low hMOF expression group (log-rank test; OS, $\mathrm{P}=0.018$ and DFS, $\mathrm{P}=0.009$ ).

\section{Discussion}

hMOF, a MYST (Moz-Ybf2/Sas3-Sas2-Tip60) family member, specifically acetylates the histone H4K16 and regulates basic physiological processes such as chromosome structure maintenance, transcriptional regulation and DNA repair $(12,13)$. In recent years, the effects of hMOF on the occurrence and development of malignant tumors has been a hot area of study in international academia. Previous studies indicated that hMOF is frequently downregulated in primary breast carcinoma and medulloblastoma (19), human renal cell (20), and colorectal carcinoma, and gastric cancer (23). However, the expression of hMOF was reportedly much higher in NSCLC compared to corresponding normal tissues, and hMOF promoted the proliferation, metastasis and adhesion of NSCLC cell lines (21). Therefore hMOF may play different roles in various malignant tumors. In the present study, the hMOF mRNA and protein 
expression in ovarian epithelial cancer tissues was much lower than that of normal ovarian tissues. The paraffin sections of different tissues from 181 patients were analyzed by immunohistochemistry (primary ovarian epithelial cancer tissues from 112 patients, borderline tumor tissues from 23 patients, benign tumor tissues from 26 patients, and normal ovarian tissues from 20 patients). The results indicated that hMOF protein expression in normal ovarian tissues and benign tumor tissues was significantly higher compared to the ovarian epithelial cancer tissues. The relationship between hMOF expression and clinicopathological data of ovarian cancer patients was further investigated and hMOF expression in stage I ovarian cancer tissues was much higher than that in stage IV ovarian cancer tissues. This finding suggests that the high expression of hMOF in ovarian cancer tissues is associated with protection from advanced ovarian cancer.

Pfister et al analyzed various factors that may affect the prognosis of medulloblastoma, and found that $\mathrm{hMOF}$ protein expression was a prognostic maker for medulloblastoma (19). However, no previous study has focused on the relationship between hMOF protein expression and ovarian cancer prognosis. To the best of our knowledge, the present study is the first to examine the relationship between hMOF protein expression and ovarian cancer prognosis. The Cox proportional-hazards regression model indicated that hMOF protein expression was an independent risk factor closely associated with ovarian cancer prognosis. In addition, the Kaplan-Meier survival analysis based on the intensity of hMOF expression found that the overall survival rate and disease-free survival rate in the high hMOF expression group was significantly higher than that in the low hMOF expression group. Thus, $h M O F$ may serve as a tumor-suppressor gene for ovarian cancer and its protein expression may be a new clinical marker that reflects ovarian cancer prognosis.

hMOF plays an important role in many aspects of processes such as repair of DNA damage, maintenance of nuclear structure and morphology, regulation of genetic transcription, apoptosis and drug resistance of cells. A decrease in the expression of hMOF may have important effects on the occurrence, development and prognosis of malignant tumors. Therefore the specific molecular mechanism responsible for the decreased expression of hMOF in this study should be further investigated. hMOF is important in the activation of ataxia telangiectasia mutated (ATM). When the cells are exposed to ionizing radiation, hMOF protein expression significantly increases and the ATM may become activated. When the $h M O F$ gene expression is decreased by siRNAs, ATM autophosphorylation and ATM kinase activity may be significantly reduced, leading to a decrease in DNA repair capacity (24). The hMOF complex contains the microporous component of the nucleus (25). Therefore, deficiencies in the expression of hMOF may affect the morphology and stability of the nuclear membrane, and convert cells into a malignant phenotype. In addition, hMOF can acetylate p53 at K120, resulting in the increased transcription of $B A X$ and $P U M A$ and cell apoptosis (26). It has been suggested that after hMOF expression decreases, multidrug-resistant cancer cells are less sensitive to the topoisomerase II inhibitors (27).

In the present study, the expression of hMOF mRNA and protein was significantly downregulated in ovarian epithelial cancer tissues, and patients with high hMOF levels showed improved survival as compared to those with low hMOF levels. Future studies should address the mechanisms hMOF may use to inhibit the development of ovarian cancer and the potential of $\mathrm{hMOF}$ as a new target for ovarian cancer treatment.

\section{Acknowledgements}

This study was supported by grants from the National Natural Science Foundation of China (nos. 81172491 and 81101527), the Education Department Doctor Project Fund (nos. 20112104110016 and 20112104120019); the Free Researcher Plan of Shengjing Hospital (no. 201303), and the Scientific Research Projects of Liaoning Province Department of Education (no. L2011129).

\section{References}

1. Strahl BD and Allis CD: The language of covalent histone modifications. Nature 403: 41-45, 2000.

2. Carrozza MJ, Utley RT, Workman JL and Côté J: The diverse functions of histone acetyltransferase complexes. Trends Genet 19: 321-329, 2003.

3. Yamada T, Mizuno K, Hirota K, et al: Roles of histone acetylation and chromatin remodeling factor in a meiotic recombination hotspot. EMBO J 23: 1792-1803, 2004.

4. Eberharter A and Becker PB: Histone acetylation: a switch between repressive and permissive chromatin. Second in review series on chromatin dynamics. EMBO Rep 3: 224-229, 2002.

5. Yang XJ: The diverse superfamily of lysine acetyltransferases and their roles in leukemia and other diseases. Nucleic Acids Res 32: 959-976, 2004.

6. Saha RN and Pahan K: HATs and HDACs in neurodegeneration: a tale of disconcerted acetylation homeostasis. Cell Death Differ 13: 539-550, 2006.

7. Ito K, Charron CE and Adcock IM: Impact of protein acetylation in inflammatory lung diseases. Pharmacol Ther 116: 249-265, 2007.

8. Gayther SA, Batley SJ, Linger L, et al: Mutations truncating the EP300 acetylase in human cancers. Nat Genet 24: 300-303, 2000.

9. Halkidou K, Gaughan L, Cook S, Leung HY, Neal DE and Robson CN: Upregulation and nuclear recruitment of HDAC1 in hormone refractory prostate cancer. Prostate 59: 177-189, 2004.

10. Yang XJ and Seto E: Lysine acetylation: codified crosstalk with other posttranslational modifications. Mol Cell 31: 449-461, 2008.

11. Hilfiker A, Hilfiker-Kleiner D, Pannuti A and Lucchesi JC: $m o f$, a putative acetyl transferase gene related to the Tip60 and MOZ human genes and to the SAS genes of yeast, is required for dosage compensation in Drosophila. EMBO J 16: 2054-2060, 1997.

12. Akhtar A and Becker PB: Activation of transcription through histone $\mathrm{H} 4$ acetylation by MOF, an acetyltransferase essential for dosage compensation in Drosophila. Mol Cell 5: 367-375, 2000.

13. Thomas T, Dixon MP, Kueh AJ and Voss AK: Mof (MYST1 or KAT8) is essential for progression of embryonic development past the blastocyst stage and required for normal chromatin architecture. Mol Cell Biol 28: 5093-5105, 2008.

14. Li X, Li L, Pandey R, et al: The histone acetyltransferase MOF is a key regulator of the embryonic stem cell core transcriptional network. Cell Stem Cell 11: 163-178, 2012.

15. Smith ER, Cayrou C, Huang R, Lane WS, Côté J and Lucchesi JC: A human protein complex homologous to the Drosophila MSL complex is responsible for the majority of histone $\mathrm{H} 4$ acetylation at lysine 16. Mol Cell Biol 25: 9175-9188, 2005.

16. Cai Y, Jin J, Swanson SK, et al: Subunit composition and substrate specificity of a MOF-containing histone acetyltransferase distinct from the male-specific lethal (MSL) complex. J Biol Chem 285: 4268-4272, 2010.

17. Taipale M, Rea S, Richter K, et al: hMOF histone acetyltransferase is required for histone $\mathrm{H} 4$ lysine 16 acetylation in mammalian cells. Mol Cell Biol 25: 6798-6810, 2005.

18. Rea S, Xouri G and Akhtar A: Males absent on the first (MOF): from flies to humans. Oncogene 26: 5385-5394, 2007. 
19. Pfister S, Rea S, Taipale M, et al: The histone acetyltransferase hMOF is frequently downregulated in primary breast carcinoma and medulloblastoma and constitutes a biomarker for clinical outcome in medulloblastoma. Int J Cancer 122: 1207-1213, 2008.

20. Wang Y, Zhang R, Wu D, et al: Epigenetic change in kidney tumor: downregulation of histone acetyltransferase MYST1 in human renal cell carcinoma. J Exp Clin Cancer Res 32: 8, 2013.

21. Zhao L, Wang DL, Liu Y, Chen S and Sun FL: Histone acetyltransferase hMOF promotes $\mathrm{S}$ phase entry and tumorigenesis in lung cancer. Cell Signal 25: 1689-1698, 2013.

22. Liu N, Zhang $\mathrm{R}$, Zhao X, et al: A potential diagnostic marker for ovarian cancer: Involvement of the histone acetyltransferase, human males absent on the first. Oncol Lett 6: 393-400, 2013.
23. Cao L, Zhu L, Yang J, et al: Correlation of low expression of hMOF with clinicopathological features of colorectal carcinoma, gastric cancer and renal cell carcinoma. Int J Oncol 44: 1207-1214, 2014.

24. Gupta A, Sharma GG, Young CS, et al: Involvement of human MOF in ATM function. Mol Cell Biol 25: 5292-5305, 2005.

25. Mendjan S, Taipale M, Kind J, et al: Nuclear pore components are involved in the transcriptional regulation of dosage compensation in Drosophila. Mol Cell 21: 811-823, 2006.

26. Sykes SM, Mellert HS, Holbert MA, et al: Acetylation of the p53 DNA-binding domain regulates apoptosis induction. Mol Cell 24: 841-851, 2006.

27. Hajji N, Wallenborg K, Vlachos P, Füllgrabe J, Hermanson O and Joseph B: Opposing effects of hMOF and SIRT1 on H4K16 acetylation and the sensitivity to the topoisomerase II inhibitor etoposide. Oncogene 29: 2192-2204, 2010. 